\title{
Comparison of imaging methods in POEMS syndrome
}

\author{
Jiri Minarika , Vlastimil Scudla ${ }^{\mathrm{a}}$, Jaroslav Bacovsky' ${ }^{\mathrm{a}}$, Tomas Pika ${ }^{\mathrm{a}}$, Filip Ctvrtlik ${ }^{\mathrm{b}}$, Iva Metelkovac, Miroslav Myslivecek ${ }^{\mathrm{c}}$
}

Background. POEMS syndrome is a clinical condition with a very heterogeneous clinical manifestation. Its presentation as well as monitoring is complex and dependent on the clinician's experience. One of the leading presenting symptoms is based on evaluation of skeletal damage with typical osteosclerotic or mixed lesions.

Aims and methods. Our aim was to compare the usefulness of different imaging methods in the diagnostics of POEMS syndrome, such as conventional radiography, densitometry, technetium scintigraphy, PET/CT scan, MRI and angiography on a series of three patients with POEMS syndrome with different clinical manifestations and course of disease. Results. Our series demonstrates different types of skeletal involvement in POEMS syndrome. Although conventional $X$-ray is the imaging method mostly used for the evaluation, its sensitivity and specificity is low. Under specific conditions, other imaging methods should be considered, giving a more complex outlook of the disease's skeletal involvement. Nevertheless, FDG-PET/CT confirmed its superiority in defining both skeletal lesions as well as the activity of the neoplastic process.

Conclusions. We conclude that the different manifestation of the disease implies the necessity of a complex evaluation of imaging methods in mutual concordance. FDG-PET/CT emerges as the most contributive method for the evaluation of both the extent and activity of the disease.

Key words: POEMS syndrome, imaging, PET/CT, conventional radiography, computed tomography, magnetic resonance

Received: June 4, 2011; Accepted: October 6, 2011; Available online: November 8, 2011 http://dx.doi.org/10.5507/bp.2011.053

\author{
${ }^{a}$ Department of Internal Medicine III - Nephrology, Rheumatology and Endocrinology, University Hospital Olomouc, Czech Republic \\ ${ }^{b}$ Department of Radiology, University Hospital Olomouc \\ 'Department of Nuclear Medicine, University Hospital Olomouc \\ Corresponding author: Jiri Minarik, e-mail: abretina@email.cz
}

\section{INTRODUCTION}

POEMS syndrome is a rare hematological disorder belonging to plasma cell dyscrasias ${ }^{1}$. It is defined as a monoclonal plasma cell disorder together with peripheral neuropathy and variable presence of other organ or tissue impairment. The acronym represents characteristic features of the syndrome $(\mathrm{P}=$ polyneuropathy, $\mathrm{O}=$ organomegaly, $\mathrm{E}=$ endocrinopathy, $\mathrm{M}=$ monoclonal gammopathy, $\mathrm{S}=$ skin lesions). There have been several attempts at the definition of diagnostic criteria of the disease. However, most of them have a limited specificity and are unable to separate the syndrome itself from the coincidence of individual symptoms. The current opinion on POEMS syndrome has been recently published as Mayo clinic criteria (Tab. 1) that reflect clinical as well as pathobiological characteristics of the syndrome ${ }^{2}$.

Unlike multiple myeloma (MM), it is usually manifested in younger patients, and its course is less aggressive ${ }^{3}$. The presence of monoclonal plasma cells and chronic progressive polyneuropathy is obligatory; however, only a few patients develop larger skeletal damage as seen in MM (ref. ${ }^{1}$ ). Moreover, the clinical manifestation can be very variable. The presence of all attributes of the acronym is sometimes quite difficult to unravel, and most patients are usually treated for other (usually neurological) disorders.
Imaging methods play a crucial role in the diagnostics as well as in the monitoring of the disease, although there is no consensus on which of the methods should be routinely used in clinical practice. Our paper presents three different manifestations of the disease with stress put on the role of imaging methods, and their contribution to the diagnostics of this clinical condition.

\section{Case report 1}

An 81 old male, originally with a neurological diagnosis of CIDP (Chronic Inflammatory Demyelinating Polyneuropathy) was presented at our hematology department in October, 1998. He had severe polyneuropathy with both axon and myelin damage (according to electromyography - EMG), and was unable to walk.

Due to the presenting symptoms we aimed our diagnostic approach from the beginning at POEMS syndrome as a majority of the findings fulfilled the diagnostic criteria: We found several skin changes - hyperpigmentation of the chest and back, hypertrichosis, thickening of the skin and several angiomas both on the body and the limbs. In his armpits there were enlarged lymph nodes, and the histobiopsy confirmed Castleman 's disease. In both the serum and spinal fluid we found monoclonal protein $\mathrm{IgG}$ lambda, and the blood count showed slight thrombocytosis $\left(485 \times 10^{9} / 1\right)$. Within the endocrinology screening, we 


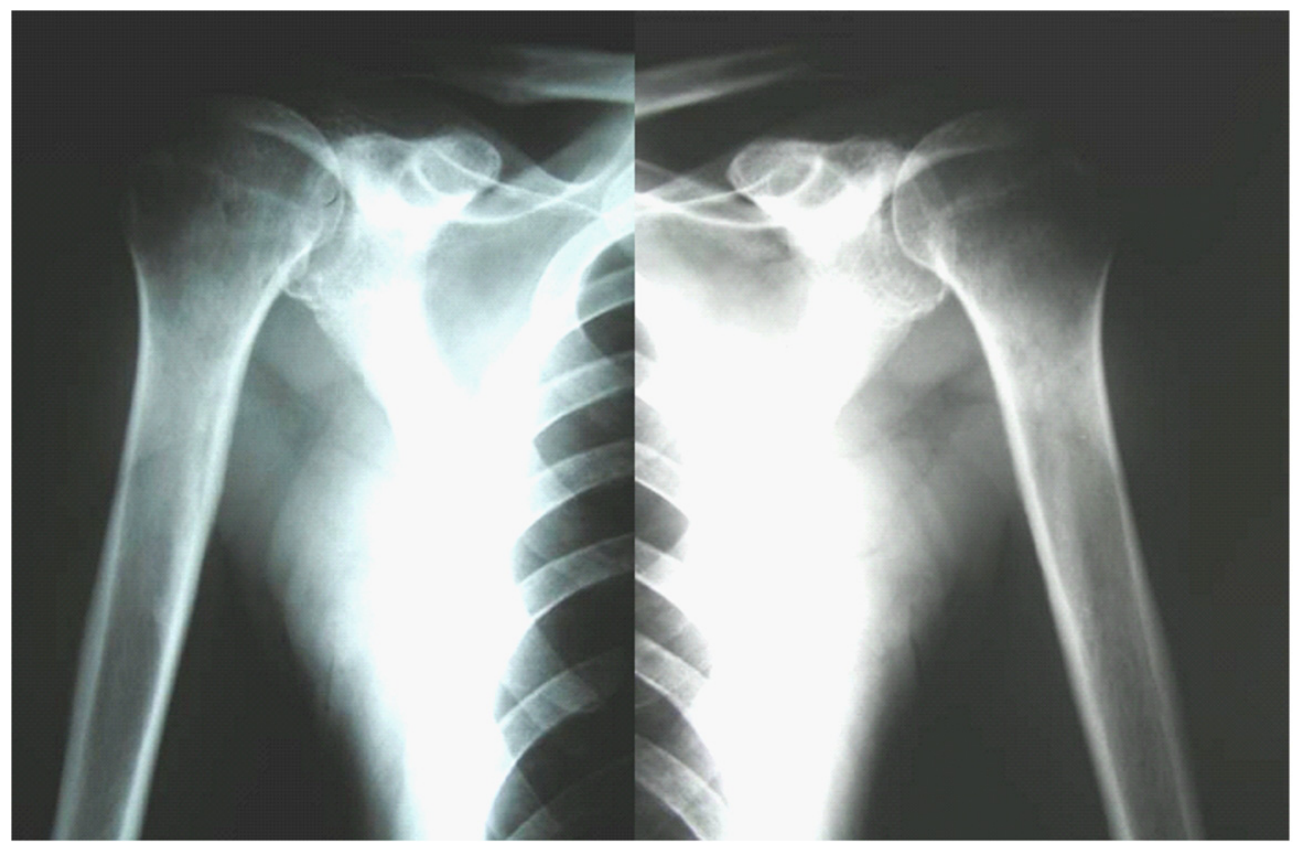

Fig. 1. Osteosclerotic "thickening" of the shaft of both proximal arm bones in a patient with POEMS syndrome.

found elevated levels of prolactin, FSH, urine cortisol, blood glucose and C-peptide. In the bone marrow there was slightly increased number of monoclonal lymphoplasmocytic elements (5.2\%). The definitive diagnosis was supported by the finding of osteosclerotic lesions, which were present on both of the proximal arm bones on the X-ray scans (Fig. 1), and the patient had higher density indices in his arms using densitometry by DEXA.

After the diagnosis we used actinotherapy for the osteosclerotic lesions and axillar lymph nodes enlargement, and conventional chemotherapy with pulses of alkylating agent (melphalan) and steroids (prednisone). The patient improved with increased muscle strength, restitution of walking and disappearance of the lymph nodes. We could trace the normalization of blood count and endocrine parameters, the decrease in monoclonal immunoglobulin and bone marrow plasma cells. The clinical assessment as well as EMG confirmed the symptoms of lower limb neuropathy, however, they remained stable with no improvement.

The course of this POEMS syndrome was quite typical with several subsequent progressions (usually defined as a slight increase in monoclonal immunoglobulin together with worsened clinical state) followed by remission of the disease. In September 2008, the patient died of an unrelated condition (unmanageable cardiac failure due to severe mitral insufficiency with valve replacement and subsequent inoperable leak).

\section{Case report 2}

A 43 years old woman was diagnosed as a CIDP (chronic inflammatory demyelinizating polyneuropathy), treated by azathioprin and prednisone. She was investigated in our department in 2004 for a small peak of monoclonal immunoglobulin IgG lambda (7.2 g/1). The presenting symptoms were, however, obscure due to several co-morbidities, although POEMS syndrome was also considered as a possible cause. The polyneuropathy was originally thought to be caused by long lasting type 2 diabetes. She had slightly enlarged liver on abdominal sonography (steatosis), and a few hemangiomas of the body and the limbs. Except for glucose metabolism (increased insulin, C-peptide), there was no pathology in the endocrine spectrum. We found no enlarged lymph nodes and the blood count as well as the biochemistry showed no significant abnormalities. In the bone marrow examination we could not find clonal plasmocytes, and there were no osteolytic nor osteosclerotic lesions on conventional X ray scan. At that time, the patient was diagnosed as having two concomitant clinical conditions - CIDP and MGUS (monoclonal gammopathy of undetermined significance).

Due to continuous worsening of the symptoms, newly diagnosed anemia and no effect of immunomodulatory therapy, the patient was re-assessed at a one year interval. At that time we found focal accumulation of clonal plasma cells in the bone marrow, and histobiopsy confirmed myeloma. The $\mathrm{X}$ ray scan was still not very persuasive with several suspect osteolytic lesions of the skull, but the crucial finding was due to conventional $99 \mathrm{mTc}$ scintigraphy (not MIBI), which found a small locus of osteoplastic remodelation in the distal sternum. The same locus (very likely hidden in the summation of the projection of spine on classical X-ray scan) was clearly visible on subsequent CT scan (Fig. 2). These findings supported our hypothesis of osteosclerotic myeloma (POEMS syndrome respectively).

The patient was treated with radiotherapy of the sternum followed by high-dose chemotherapy and autologous stem cell transplantation. Chemotherapy led to the normalization of blood count, a decrease in monoclonal immunoglobulin and bone marrow plasma cell involvement. The neuropathy of the limbs remained unimproved, apparently due to the presence of concomitant diabetic neuropathy. 


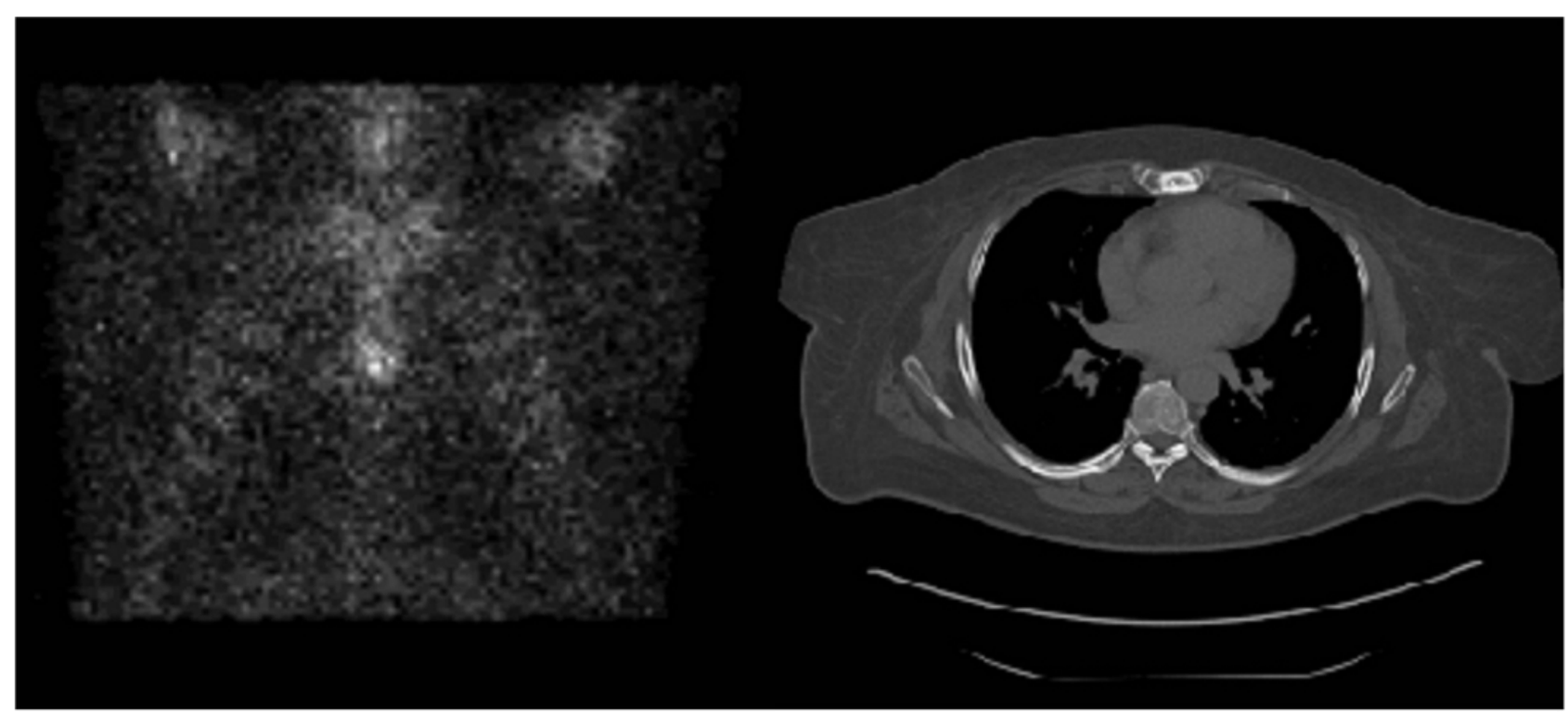

Fig. 2. Osteosclerotic lesion in distal sternum of a patient with POEMS syndrome. Focal accumulation of $99 \mathrm{mTc}$ on conventional technetium scintigram (left). Osteosclerosis visible as "thickening" of sternum on CT scan (right).

The course of the disease was characterized by two subsequent progressions followed by conventional chemotherapy regimens (melphalan plus prednisone and cyclophosphamide plus dexamethasone) with no significant change in overall patient status. The duration of the disease remission was about 9-12 months. At present, the patient is progressing with the eruption of multiple skin hemangiomas and an increase of paraprotein. She is being treated with chemotherapy using lenalidomide and prednisone.

\section{Case report 3}

A 25 year old man with an 8-month history of polyradiculoneuritis was treated with azathioprin and methylprednisolone in the neurological department. $\mathrm{He}$ temporarily improved and started rehabilitation with the interruption of immunosuppressive treatment. This treatment-free period was complicated within 3 months by the development of polyserositis with lower limb edema, pleural and cardiac effusions, ascites and paraparesis of lower limbs, still with no pain or signs of bone damage. He lost $25 \mathrm{~kg}$ in 8 months and developed overall weakness

Table 1. Diagnostic criteria of POEMS syndrome - Mayo clinic criteria from 2007(ref. ${ }^{2}$ )

\begin{tabular}{lll}
\hline Major criteria & \multicolumn{1}{c}{ "Mayo clinic criteria 2007"* } & \\
\hline Polyneuropathy & $\begin{array}{l}\text { Organomegaly (splenomegaly, } \\
\text { hepatomegaly, adenomegaly) }\end{array}$ & Clubbing \\
Monoclonal gammopathy & $\begin{array}{l}\text { Edema, pleural effusion, ascites } \\
\text { Endocrinopathy (adrenal, thyroid, } \\
\text { Sclerotic bone lesions }\end{array}$ & Weight loss \\
pancreatic) & Hyperhidrosis \\
Castleman disease & $\begin{array}{l}\text { Skin changes (hyperpigmentation, } \\
\text { hypertrichosis, hemangioms, flush- } \\
\text { ing, acrocyanosis, white nails) }\end{array}$ & $\begin{array}{l}\text { Pulmonary hypertension/restrictive } \\
\text { lung disease }\end{array}$ \\
$\begin{array}{ll}\text { Elevation of Vascular Endothelial } \\
\text { Growth Factor (VEGF) }\end{array}$ & $\begin{array}{l}\text { Papilledema } \\
\text { Thrombocytosis or polycytemia }\end{array}$ & Low vitamin $\mathrm{B}_{12}$ level \\
\hline
\end{tabular}

\footnotetext{
* Polyneuropathy with monoclonal gammopathy or Castleman disease must be present in all patients. For the diagnosis at least one more major and one minor criterion is required.
} 


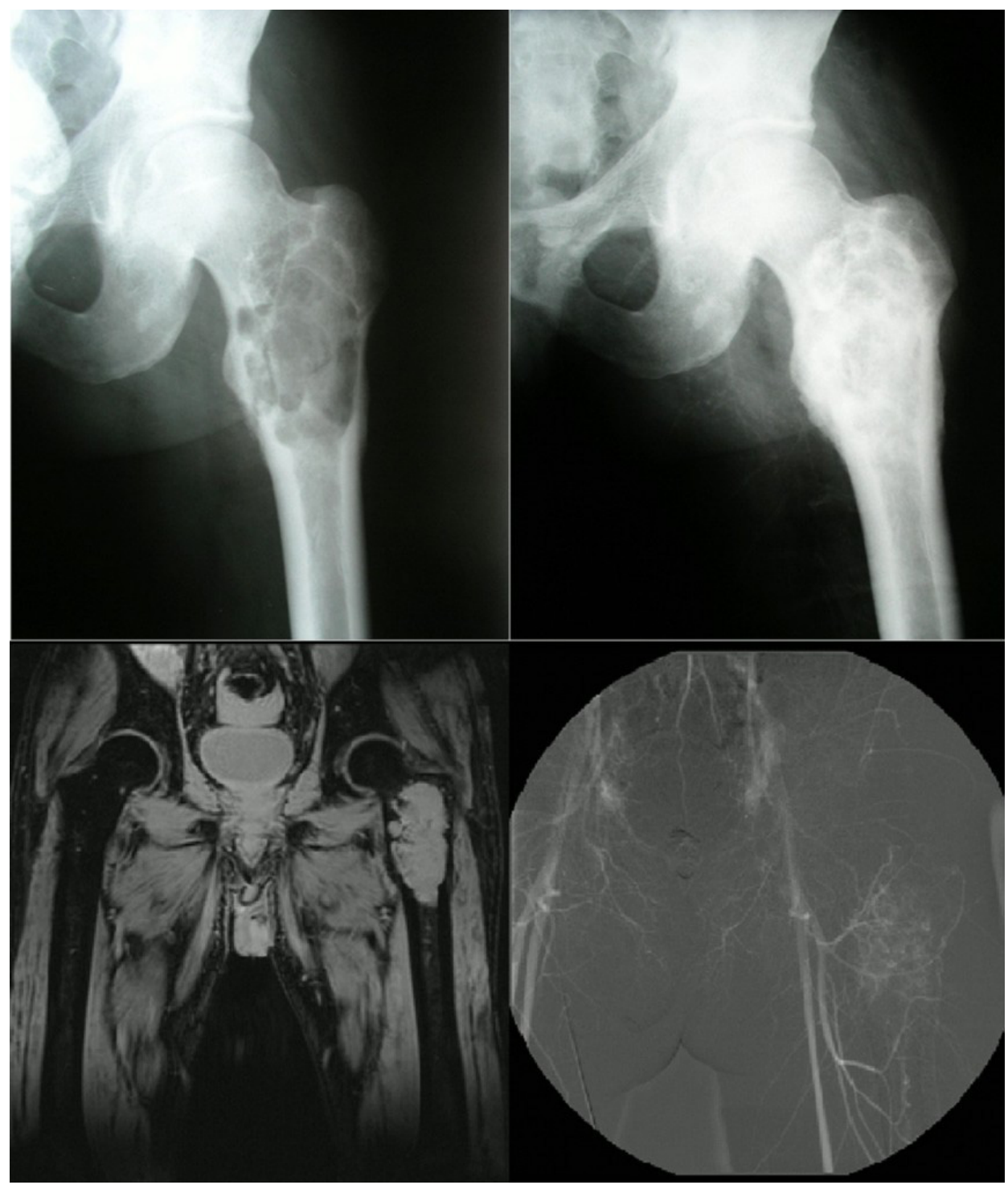

Fig. 3. Large osteolytic lesion with osteosclerotic rims and pathological vascularisation in a patient with POEMS syndrome.

Conventional radiography presents large osteolytic tumor of proximal femur (A) with subsequent recalcification after successful therapy (B). The tumor consists of mixed osteolytic and osteosclerotic involvement seen on MRI (C), and has pathological vascularisation visible on digital subtraction angiography (D).

and dyspnea. The initial screening for possible infectious, malignant or systemic cause was, however, negative, with normal biochemistry including CRP, negative infectious disease screening, normal oncomarkers, immunological screening and endocrine tests (repeatedly). He had only substantial thrombocytosis $\left(1016 \times 10^{9} / 1\right)$ and the presence of monoclonal immunoglobulin IgG lambda (up to $10 \mathrm{~g} / 1$ ). Ultrasonography confirmed ascites, mild hepatomegaly, pleural and cardiac effusions with no other pathology on parenchymatous organs. Tests done on the aspirate of both pleural effusion and ascites showed transudate with no biochemical or cytological pathology, assessment of liquor was negative as well as the result of bone marrow trephine biopsy including immunophenotyping.

Examination of bone X-ray (suspicion of myeloma) showed a large cystic lesion $10 \times 5 \mathrm{~cm}$ in the left proximal femur with MRI verified mixed osteolytic and osteosclerotic involvement and pathological vascularisation on angiography (Fig. 3). The most contributive approach was FDG-PET/CT which unraveled multiple mixed os- teolytic/osteosclerotic lesions with FDG activity on clavicles, vertebral bodies and ribs. The definitive diagnosis was confirmed by the finding of a compact infiltrate of monoclonal plasmocytes from targeted biopsy of one of the larger lesions (right clavicle).

After successful diagnosis the treatment started with radiotherapy of the hip, systemic chemotherapy (cyclophosphamide and dexamethasone), and preparation for autologous transplant. The stem cell harvest was, however, unsuccessful (twice in a 2 month interval), probably due to long-term non-specific pretreatment. The following therapy consisted of conventional drugs and led to the normalization of laboratory findings, to the disappearance of effusions and improvement of overall condition including substantial regression of peripheral neuropathy, recalcification of the osteolytic lesions (Fig. 3), and restitution of activities of daily living including normal walking.

The patient is at the moment in remission with no chemotherapy. 


\section{DISCUSSION}

POEMS syndrome represents a very heterogeneous disease with variable presence of accompanying symptoms. Except for characteristic polyneuropathy and monoclonal gammopathy, the other associated symptoms might not be evident at the onset of the disease. Imaging methods are therefore very useful in the diagnostics as well as in the assessment of bone involvement in POEMS syndrome. Still, there is no standard algorithm for choice of specific method, and the usefulness of different approaches varies throughout the literature.

Conventional radiography might be contributive: However, the bone lesions can be confused with other conditions such as bone cysts, nonossifying fibromas, benign bone islands or fibrous dysplasia ${ }^{1}$. There are two types of skeletal manifestations in POEMS syndrome: proliferative changes and focal bone lesions ${ }^{4}$. Although approximately $95 \%$ of patients with POEMS syndrome have osteosclerotic lesions, some of the lesions can be lytic with a sclerotic rim or mixed with different proportion of sclerotic and lytic involvement ${ }^{1}$. Focal lesions can be solitary or multiple, usually found in the axial bones ${ }^{4}$. The present paper accounts for all the manifestations - as a "solitary osteosclerotic lesion" in case report 2 (Fig. 2), "oligo-focal" proliferative changes in case report 1 (Fig. 1), and "multiple mixed osteolytic lesions with osteosclerotic component" in case report 3 (Fig. 3).

The extent of the lesions does not necessarily correspond to the severity of the disease but the presence of bone involvement is pathognomonic. Interestingly, we found increased bone density in the areas with osteosclerotic involvement, which contributed to the evaluation of the lesion. DEXA evaluation might therefore be an inexpensive complementary imaging method, although contributive only in selected cases due to the character and variable localization of sclerotic lesions.

There have been just a few reports on the contribution of MRI in the diagnostics of POEMS syndrome ${ }^{5-9}$. The MRI imaging in POEMS syndrome is quite rare as the diagnosis is usually based on clinical symptoms, and the MRI scans are mostly performed in situations of diagnostic uncertainty. On the other hand, the character on T1 and T2-weighted sequences strongly illustrates the different types of bone involvement ${ }^{5}$.

The contribution of angiography is dubious as the lesions are usually not of such an extent as seen in case report 3. On the other hand, a recent studies of POEMS syndrome that focus on the role of angiogenic cytokines, especially the vascular endothelial growth factor (VEGF) confirmed their elevated expression in POEMS syndrome, and their possible responsibility for the onset of the disease $\mathrm{e}^{1,10-13}$. From this point of view, a search for increased neovascularisation could be challenging in the assessment of the extent of disease even before actual bone damage.

The osteosclerotic lesions are usually referred with negative radionuclide bone scan, although some previous reports have registered local increased uptake of radiotracer ${ }^{4,14-16}$, usually attributed to focal cortical expansion, periostitis and sclerotic reaction ${ }^{14}$. Similarly, in case report
2 we presented a patient with positive bone scintigraphy based on which the diagnostic discretion was aimed at this clinical condition.

CT scan is more informative, although still not routinely used for whole body assessment. We found just two recent papers which demonstrated the potential of FDG-PET/CT scan in the evaluation of POEMS (ref. ${ }^{17,18}$ ). Although quite expensive, PET/CT seems to be a very convenient method for detection of the activity as well as the extent and localization of bone and/or lymph node lesions. Also, our findings in case report 3 support the use of PET/CT especially in patients with diagnostic complexity. Despite the negative laboratory findings and missing clinical symptoms of bone damage, PET/CT unraveled vast skeletal damage and also extraskeletal localization of the disease.

The present paper aims to demonstrate the complexity of imaging in POEMS syndrome. As the condition is quite rare, there are no recommendations on which of the imaging methods are the most suitable. The assessed groups of patients are usually too small for generalization. Still, the heterogeneity and different manifestation of the disease foreshadow the necessity of a complex evaluation of different imaging methods in mutual concordance. We also show the increasing role of FDG-PET/CT which might have the ability to reflect the extent of POEMS syndrome, especially in X-ray negative patients, as well as the possible extra-osseal localization of the disease.

\section{AKNOWLEDGEMENT}

Supported by VVZ MSM 6198959205.

\section{REFFERENCES}

1. Dispenzieri A. Diagnosis and treatment of POEMS syndrome. In Rajkumar SV, Kyle RA, Treatment of multiple myeloma and related disorders. Cambridge university press, New York, NY, USA, 2009: 182195. ISBN 13: 9780521515030.

2. Dispenzieri A. Mayo Clinic Criteria for the Diagnosis of Polyneuropathy, Organomegaly, Endocrinopathy, Monoclonal Gammopathy, and Skin Changes Syndrome. Note. From "POEMS Syndrome". Blood Reviews 2007,21:287.

3. Rajkumar SV, Kyle RA, Suarez GA, Dispenzieri A. Neuropathy associated with plasma cell proliferative disorders. In Gertz MA, Greipp PR. Multiple myeloma and related plasma cell disorders. Mayo Foundation for medical education and research, Rochester, MN, USA, 2004:35-52. ISBN 3-540-00811-X.

4. Narvaez JA, Majos C, Narvaez J, Valls C, Fernandez-Cabrera L. POEMS syndrome: unusual radiographic, scintigraphic and CT features. Eur Radiol 1998,8:134-6.

5. Chong ST, Beasley HS, Daffner RH. POEMS syndrome: radiographic appearance with MRI correlation. Skeletal Radiol, 2006,35:690-695.

6. Michel JL, Gaucher-Hugel AS, Reynier C, Lhoste A, Philippe P. POEMS syndrome: imaging of skeletal manifestations, a study of 8 cases. J Radiol 2003,84:393-7.

7. Kim JW, Lee SK, HA KM, Kim KH, Joh GY, Kim HJ, Yang SO. POEMS syndrome: a case report. J Korean Med Sci 1992;7:79-84.

8. Furuzono H, Moritoyo T, Yamada H, Sugihara R, Nagamatsu K. A case of Crow-Fukase syndrome which developed seven years following myelopathy of unknown origin. Rinsho Shinkeigu 1993;33:56-60.

9. Brazis PW, Liesegang TJ, Bolling JP, Kashii S, Trachtman M, Burde RM When do optic disc edema and peripheral neuropathy constitute poetry? Surv Ophthalmol 1990;35:219-25. 
10. Watanabe $\mathrm{O}$, Maruyama I, Arimura K, Kitajima I, Arimura H, Hanatan M, Matsuo K, Arisato T,Osame M. Overproduction of vascular endothelial growth factor/vascular permeability factor is causative in Crow-Fukase (POEMS) syndrome. Muscle Nerve 1998;21:1390-7.

11. Scarlato M, Previtali SC, Carpo M, Pareyson D, Briani C, Del Bo R Nobile-Orazio E, Quattrini A, Comi GP. Polyneuropathy in POEMS syndrome: role of angiogenic factors in the pathogenesis. Brain 2005,128:1911-20.

12. Soubrier M, Dubost JJ, Serre AF, Ristori JM, Sauvezie B, Cathebras P, Piette JC, Chapman A, Authier FJ, Gherardi RK. Growth factors in POEMS syndrome: evidence for a marked increase in circulating vascular endothelial growth factor. Arhritis Rheum 1997,40:786-7.

13. Gherardi RK, Belec L, Soubrier M, Malapert D, Zuber M, Viard JP. Overproduction of proinflammatory cytokines imbalanced by their antagonists in POEMS syndrome. Blood 1996,87:1458-65.
14. Bessler W, Antonucci F, Stamm B, Stuckmann G, Vollrath T. Case report 646. POEMS syndrome. Skeletal Radiol 1991,20:212-5.

15. Mertens I, Vandeputte L, Van Haecke P, Thomas J, Samson I, Lateur L, De Wolf-Peeters C, Paridaens R. Sclerotic IgA myeloma and polyneuropathy: The POEMS syndrome. Ann Oncol 1995,6:731-2.

16. Jin S-A,Baek S-W, Song I-Ch,Yun G-W,Yang Y-J,Lee H-J,Yun H-J, Kim J-M, Jo D-Y,Kim S. A case of multiple myeloma associated with multifocal osteosclerosis (multiple myeloma with osteosclerosis).Korean J Hematol 2009,44:188-92.

17. Alberti MA, Martinez-Yelamos S, Fernandez A, Vidaller A, Narvaez JA, Cano LM, Gamez C, Martinez Matos JA. 18-F-FDG PET/CT in the evaluation of POEMS syndrome. Eur J Radiol 2010,76:180-2.

18. An YS, Yoon JK, Hong SP, Joh CW, Yoon SN. 18F-FDG PET/CT in POEMS Syndrome. Nucl Med Mol Imaging 2007,41:66-7. 